\title{
ACTIONS OF COCOMMUTATIVE HOPF ALGEBRAS
}

\author{
MARTIN LORENZ, BACH NGUYEN, AND RAMY YAMMINE
}

\begin{abstract}
Let $H$ be a cocommutative Hopf algebra acting on an algebra $A$. Assuming the base field to be algebraically closed and the $H$-action on $A$ to be integral, that is, it is given by a coaction of some Hopf subalgebra of the finite dual $H^{\circ}$ that is an integral domain, we stratify the prime spectrum Spec $A$ in terms of the prime spectra of certain commutative algebras. For arbitrary $H$-actions in characteristic 0 , we show that the largest $H$-stable ideal of $A$ that is contained in a given semiprime ideal of $A$ is semiprime as well.
\end{abstract}

\section{INTRODUCTION}

0.1. Let $H$ be a Hopf algebra over a field $\mathbb{k}$ and let $A$ be an arbitrary associative $\mathbb{k}$-algebra. An action of $H$ on $A$ is given by a k-linear map $H \otimes A \rightarrow A, h \otimes a \mapsto h . a$, that makes $A$ into a left $H$-module and satisfies the "measuring" conditions $h .(a b)=\left(h_{1} . a\right)\left(h_{2} . b\right)$ and $h .1=\langle\varepsilon, h\rangle 1$ for $h \in H$ and $a, b \in A$. Here, $\otimes=\otimes_{\mathbb{k}}, \Delta h=h_{1} \otimes h_{2}$ denotes the comultiplication of $H$, and $\varepsilon$ is the counit. We will write $H C A$ to indicate such an action. Algebras equipped with an $H$-action are called left $H$-module algebras. With algebra maps that are also $H$-module maps as morphisms, left $H$-module algebras form a category, ${ }_{H} \mathrm{Alg}$.

For example, an action of a group algebra $\mathbb{k} G$ on $A$ amounts to the datum of a group homomorphism $G \rightarrow$ Aut $A$, the automorphism group of the algebra $A$. For the enveloping algebra $U \mathfrak{g}$ of a Lie $\mathbb{k}$-algebra $\mathfrak{g}$, an action $U \mathfrak{g} \subset A$ is given by a Lie homomorphism $\mathfrak{g} \rightarrow$ Der $A$, the Lie algebra of all derivations of $A$. In both these prototypical cases, the acting Hopf algebra is cocommutative. This article investigates the effect of a given action $H C A$ of an arbitrary cocommutative Hopf algebra $H$ on the prime and semiprime ideals of $A$, partially generalizing prior work of the first author on rational actions of algebraic groups [11], [12], [13]. The interesting article [18] by Skryabin covers related territory, but the actual overlap with our work is insubstantial.

0.2. For now, let $H$ be arbitrary and let $A \in{ }_{H}$ Alg. An ideal $I$ of $A$ that is also an $H$ submodule of $A$ is called an $H$-ideal. The action $H C A$ then passes down to an $H$-action on the quotient algebra $A / I$. The sum of all $H$-ideals that are contained in an arbitrary ideal $I$, clearly the unique largest $H$-ideal of $A$ that is contained in $I$, will be referred to as the $H$-core of $I$ and denoted by $I: H$. Explicitly,

$$
I: H=\{a \in A \mid H . a \subseteq I\} .
$$

If $A \neq 0$ and the product of any two nonzero $H$-ideals of $A$ is again nonzero, then $A$ is said to be $H$-prime. An $H$-ideal $I$ of $A$ is called $H$-prime if $A / I$ is $H$-prime. It is easy to see

2010 Mathematics Subject Classification. 16T05, 16T20.

Key words and phrases. Hopf algebra, action, quantum invariant theory, prime spectrum, stratification, prime ideal, semiprime ideal, integral action, rational action, algebraic group, Lie algebra, derivation. 
that $H$-cores of prime ideals are $H$-prime. Denoting the collection of all $H$-primes of $A$ by $H$-Spec $A$, we thus obtain a map $\operatorname{Spec} A \rightarrow H$-Spec $A, P \mapsto P: H$. The fibers

$$
\operatorname{Spec}_{I} A \stackrel{\text { def }}{=}\{P \in \operatorname{Spec} A \mid P: H=I\}
$$

are called the $H$-strata of $\operatorname{Spec} A$. The stratification $\operatorname{Spec} A=\bigsqcup_{I \in H \text {-Spec } A} \operatorname{Spec}_{I} A$ was pioneered by Goodearl and Letzter [7] in the case of group actions or, equivalently, actions of group algebras. It has proven to be a useful tool for investigating $\operatorname{Spec} A$, especially for rational actions of a connected affine algebraic group $G$ over an algebraically closed field $\mathbb{k}$. In this case, one has a description of each stratum $\operatorname{Spec}_{I} A$ in terms of the prime spectrum of a suitable commutative algebra [12, Theorem 9]. Our principal goal is to generalize this result to the context of cocommutative Hopf algebras. This is carried out in Section 3 of this article. The first two sections serve to deploy some generalities on actions of Hopf algebras that are needed for the proof, with Section 2 focusing on the cocommutative case.

0.3. To state our main result, we make the following observations; see Sections 1-3 for details. Let $H$ be cocommutative and $\mathbb{k}$ algebraically closed. Assume that the action $H C A$ is locally finite, that is, $\operatorname{dim}_{\mathbb{k}} H . a<\infty$ for all $a \in A$. Then $A$ becomes a right comodule algebra over the (commutative) finite dual $H^{\circ}$ of $H$ :

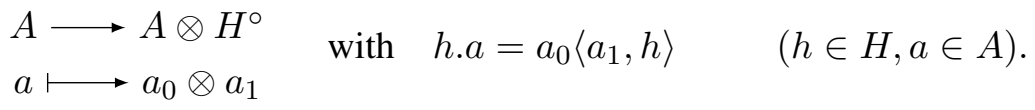

The action $H \subset A$ will be called integral if the image of the map (2) is contained in $A \otimes \mathcal{O}$ for some Hopf subalgebra $\mathcal{O} \subseteq H^{\circ}$ that is an integral domain. This condition serves as a replacement for connectedness in the case of algebraic group actions. Assuming it to be satisfied, it follows that each $I \in H$-Spec $A$ is in fact a prime ideal of $A$. Consequently, the extended center $\mathcal{C}(A / I)=\mathcal{Z} \mathrm{Q}(A / I)$ is a k-field, where $\mathrm{Q}(A / I)$ denotes the symmetric ring of quotients of $A / I$. The action $H C A / I$ extends uniquely to an $H$-action on $\mathrm{Q}(A / I)$ and this action stabilizes the center $\mathcal{C}(A / I)$. Furthermore, $\mathcal{O} \in{ }_{H} \mathrm{Alg}$ via the "hit" action $\rightarrow$ that is given by $\langle h \rightarrow f, k\rangle=\langle f, k h\rangle$ for $f \in \mathcal{O}$ and $h, k \in H$. The actions $\rightarrow$ and $H \subset \mathcal{C}(A / I)$ combine to an $H$-action on the tensor product; so

$$
C_{I}:=\mathcal{C}(A / I) \otimes \mathcal{O} \in{ }_{H} \mathrm{Alg} .
$$

The algebra $C_{I}$ is a commutative integral domain. We let $\operatorname{Spec}^{H} C_{I}$ denote the subset of Spec $C_{I}$ consisting of all prime $H$-ideals of $C_{I}$.

Theorem 1. Let $H$ be a cocommutative Hopf algebra over an algebraically closed field $\mathbb{k}$ and let $A$ be a $\mathbb{k}$-algebra that is equipped with an integral action $H \subset A$. Then, for any $I \in H$-Spec $A$, there is a bijection

$$
c: \operatorname{Spec}_{I} A \stackrel{\sim}{\longrightarrow} \operatorname{Spec}^{H} C_{I}
$$

having the following properties, for any $P, P^{\prime} \in \operatorname{Spec}_{I} A$ :

(i) $c(P) \subseteq c\left(P^{\prime}\right)$ if and only if $P \subseteq P^{\prime}$, and

(ii) $\operatorname{Fract}\left(C_{I} / c(P)\right) \cong \mathcal{C}((A / P) \otimes \mathcal{O})$. 
0.4. As a first example, let $G$ be an affine algebraic $\mathbb{k}$-group and let $\mathcal{O}=\mathcal{O}(G)$ be the algebra of polynomial functions on $G$. Then $\mathcal{O} \subseteq H^{\circ}$ with $H=\mathbb{k} G$. A rational $G$-action on $A$, by definition, is a locally finite action $H \subset A$ such that the image of (2) is contained in $A \otimes \mathcal{O}$. If $G$ is connected, then $\mathcal{O}$ is an integral domain and so the action is integral. In this setting, Theorem 1 is covered by [12, Theorem 9].

Next, let $\mathfrak{g}$ be a Lie $\mathbb{k}$-algebra acting by derivations on $A$ and assume that every $a \in A$ is contained in some finite-dimensional $\mathfrak{g}$-stable subspace of $A$. With $H=U \mathfrak{g}$, we then have a locally finite action $H C A$ and hence a map (2). If char $\mathbb{k}=0$, then the convolution algebra $H^{*}$ is a power series algebra over $\mathbb{k}$ and hence $H^{*}$ is a commutative domain; see $\S 4.3$ below. Since $H^{\circ}$ is a subalgebra of $H^{*}$, we may take $\mathcal{O}=H^{\circ}$ and so we have an integral action. Theorem 1 appears to be new in this case.

0.5. In Section 4 , we show that if char $\mathbb{k}=0$ and $H$ is cocommutative, then the core operator .:H preserves semiprimess. Recall that an ideal $I$ of $A$ is called semiprime if $I$ is an intersection of prime ideals or, equivalently, $A / I$ has no nonzero nilpotent ideals. In Section 4, we turn to the question as to whether $I: H$ is then semiprime as well. This question may be reformulated in various alternative ways (Lemma 15). In general, the answer is negative: even for a cocommutative Hopf algebra $H$, semiprimeness may be lost upon passage to the $H$-core. For instance, consider the group algebra $A=\mathbb{k} G$ with its standard $G$-grading. For $G$ finite, this grading amounts to an action of the Hopf dual $H=(\mathbb{k} G)^{*}$ on $A$. The only $H$-ideals of $A$ are 0 and $A$; so the $H$-core of every proper ideal of $A$ is 0 . If $G$ is abelian, then $H$ is cocommutative. If the operator.$: H$ is to preserve semiprimeness for any such $G$, then we must require that char $\mathbb{k}=0$ by Maschke's Theorem. It turns out that this is also sufficient in general:

Theorem 2. Let $A \in{ }_{H} \mathrm{Alg}$ and assume that $H$ is cocommutative and char $\mathbb{k}=0$. Then $I: H$ is semiprime for every semiprime ideal I of $A$.

0.6. In future work, we hope to pursue the general theme of this article for Hopf algebras that are not necessarily cocommutative. In particular, we plan to address "rationality" of prime ideals and explore the Dixmier-Mœglin equivalence in the context of Hopf algebra actions, generalizing the work on group actions in [11], [12].

Notations and conventions. We work over a base field $\mathbb{k}$ and continue to write $\otimes=\otimes_{\mathbb{k}}$. Throughout, $H$ is a Hopf $\mathbb{k}$-algebra, cocommutative when so specified. The counit and the antipode of $H$ will be denoted by $\varepsilon$ and $\mathrm{S}$, respectively. Furthermore, we fix the following notations for the remainder of this article:

$A$ will be a left $H$-module algebra, with $H$-action $h \otimes a \mapsto h . a(h \in H, a \in A)$; $A^{H}=\{a \in A \mid h . a=\langle\varepsilon, h\rangle a$ for all $h \in H\}$ is the subalgebra of $H$-invariants; $B=\operatorname{Hom}_{\mathbb{k}}(H, A)$ will denote the convolution algebra.

\section{Generalities on ACtions}

1.1. The convolution algebra $B$. We begin by introducing certain subalgebras and automorphisms of $B$ as well as some $H$-operations on $B$ that will be used throughout this article.

First, the counit of $H$ and the action $H C A$ give rise to the following maps $\iota, \delta: A \rightarrow B$, which are easily seen to be $\mathbb{k}$-algebra embeddings:

$$
\iota a=(h \mapsto\langle\varepsilon, h\rangle a) \quad \delta a=(h \mapsto h . a) \quad(h \in H, a \in A) .
$$


We will generally identify $A$ with $\iota A$ and we will also identify the linear dual $H^{*}$ with the image of the algebra map $u_{*}: H^{*} \hookrightarrow B$ that is given by the unit $u: \mathbb{k} \rightarrow A$. In this way, we view $A, H^{*}$, and $A \otimes H^{*}$ as $\mathbb{k}$-subalgebras of $B$, with $A \otimes H^{*}$ consisting of all k-linear maps $H \rightarrow A$ that have finite rank. Explicitly, $a \otimes f=(h \mapsto a\langle f, h\rangle), \iota a=a \otimes \varepsilon$ and $u_{*} f=1 \otimes f$ for $a \in A, f \in H^{*}$.

The algebra $B$ becomes a left $H$-module algebra via the $\rightarrow$-action, which is defined by

$$
(h-b)(k)=b(k h) \quad(h, k \in H, b \in B) .
$$

We will write $(B, \rightarrow)$ when viewing $B \in{ }_{H}$ Alg with (4). The map $\delta: A \rightarrow(B, \rightarrow)$ is a morphism in ${ }_{H} \mathrm{Alg}$ and $A \otimes H^{*}$ is an $H$-module subalgebra of $(B, \rightarrow)$.

Our main focus later will be on the following alternative left $H$-module structure on $B$, which takes into account the given action $H C A$ :

$$
(h \cdot b)(k)=h_{1} \cdot b\left(k h_{2}\right) .
$$

If $H$ is cocommutative, then $B \in{ }_{H} \mathrm{Alg}$ with (5) as well; see Proposition 6 below. In general, we may pass between (4) and (5) by means of the k-linear automorphisms $\Phi, \Psi: B \stackrel{\sim}{\sim} B$ that are defined by

$$
\Phi b=\left(h \mapsto h_{1} \cdot b\left(h_{2}\right)\right) \quad \text { and } \quad \Psi b=\left(h \mapsto \mathrm{S}\left(h_{1}\right) \cdot b\left(h_{2}\right)\right) .
$$

These maps are inverse to each other and they satisfy the following "intertwining" formulas, for any $a \in A, b \in B$ and $h \in H$ :

$$
\begin{gathered}
\Phi((\iota a) b)=\delta a \Phi b, \quad \Psi((\delta a) b)=\iota a \Psi b \\
\Phi(h \cdot b)=h-(\Phi b), \quad h \cdot(\Psi b)=\Psi(h-b) .
\end{gathered}
$$

The $\Psi$-identities follow from those for $\Phi=\Psi^{-1}$. To verify (6), one checks that both sides send a given $h \in H$ to $h_{1} \cdot\left(a b\left(h_{2}\right)\right)$. For (7), observe that $(\Phi b)(h)=(h \cdot b)(1)$ and so $\Phi(h \cdot b)(k)=(k h \cdot b)(1)=(\Phi b)(k h)=(h-(\Phi b))(k)$ for $h, k \in H$ and $b \in B$. Finally, note that $\Phi$ and $\Psi$ fix the unit element $1_{B}=u_{*}(\varepsilon)$ and they are right linear for the subalgebra $\operatorname{Hom}_{\mathbb{k}}\left(H, A^{H}\right) \subseteq B$. In particular, $\Phi$ and $\Psi$ restrict to the identity map on $H^{*}$ and are right $H^{*}$-linear.

1.2. Invariants and the locally finite part of $(B,-)$. The locally finite part of any $H$ module algebra $A$ is defined by

$$
\begin{aligned}
A_{\mathrm{fin}}: & =\left\{a \in A \mid \operatorname{dim}_{\mathbb{k}} H . a<\infty\right\} \\
& =\{a \in A \mid I . a=0 \text { for some cofinite ideal } I \text { of } H\} .
\end{aligned}
$$

Here, "cofinite" is short for "having finite codimension." The locally finite part $A_{\text {fin }}$ is always an $H$-module subalgebra of $A$ containing the algebra of invariants, $A^{H}$.

The following lemma determines the invariants and the locally finite part of $(B, \rightarrow)$.

Lemma 3. (a) $(B, \rightarrow)^{H}=\iota A \cong A$;

(b) $(B, \rightarrow)_{\text {fin }}=\{b \in B \mid$ Ker $b$ contains some cofinite ideal of $H\} \cong A \otimes H^{\circ}$.

Proof. (a) Note that $b \in(B, \rightarrow)^{H}$ if and only if $b(k h)=\langle\varepsilon, h\rangle b(k)$ for all $h, k \in H$. Equivalently, $b(h)=\langle\varepsilon, h\rangle b(1)$ for all $h \in H$, which in turn states that $b=\iota(b(1))$. The assertion about $H$-invariants follows. 
(b) For any ideal $I$ of $H$ and any $b \in B$, the equality $I \rightarrow b=0$ is equivalent to $I \subseteq$ $\operatorname{Ker} b$; so $b \in(B, \rightarrow)_{\text {fin }}$ if and only if Ker $b$ contains some cofinite ideal of $H$. In particular, $\iota A \subseteq(B, \rightarrow)_{\text {fin }}$ and the embedding $u_{*}: H^{*} \hookrightarrow B$ sends the finite dual $H^{\circ}$ to $(B, \rightarrow)_{\text {fin }}$. The isomorphism between the subalgebra of $B$ consisting of all finite-rank maps and $A \otimes H^{*}$ (§1.1) restricts to an isomorphism of subalgebras, $(B, \rightarrow)_{\text {fin }} \cong A \otimes H^{\circ}$.

The isomorphisms in Lemma 3 will be treated as identifications as in $\S 1.1$. In particular, we will write $A \otimes H^{\circ}=(B, \rightarrow)_{\text {fin }}$ and identify $A$ and $H^{\circ}$ with the subalgebras $\iota A=A \otimes \varepsilon$ and $u_{*} H^{\circ}=1 \otimes H^{\circ}$, respectively. As was mentioned, $(B, \rightarrow)_{\text {fin }}$ is an $H$-module subalgebra of $(B,-)$; explicitly, with $b=a \otimes f\left(a \in A, f \in H^{\circ}\right)$ formula (4) becomes

$$
h \rightarrow(a \otimes f)=a \otimes(h-f)=a \otimes f_{1}\left\langle f_{2}, h\right\rangle .
$$

Furthermore, $(B,-)_{\text {fin }}=A \otimes H^{\circ}$ is also stable under the $H$-operation (5), which becomes the standard Hopf operation on tensor products:

$$
h \cdot(a \otimes f)=h_{1} \cdot a \otimes\left(h_{2}-f\right)=h_{1} \cdot a \otimes f_{1}\left\langle f_{2}, h_{2}\right\rangle .
$$

More generally, for any Hopf subalgebra $\mathcal{O} \subseteq H^{\circ}$, we will consider the subalgebra

$$
A_{\mathcal{O}}:=A \otimes \mathcal{O} \subseteq(B, \rightarrow)_{\text {fin }} .
$$

Each such $A_{\mathcal{O}}$ is stable under both $\rightarrow$ and $\cdot$ by (8) and (9).

Lemma 4. Let $\mathcal{O} \subseteq H^{\circ}$ be a Hopf subalgebra. The $\mathbb{k}$-subspaces of $A_{\mathcal{O}}$ that are stable under right multiplication by $\mathcal{O}$ and under the $\rightarrow$-action (8) are exactly those of the form $W \otimes \mathcal{O}$, where $W$ is an arbitrary $\mathbb{k}$-subspace of $A$.

Proof. Certainly, each $W \otimes \mathcal{O}$ is stable under right multiplication by $\mathcal{O}$ and under (8). For the converse, equip $A_{\mathcal{O}}$ with the "trivial" right $\mathcal{O}$-Hopf module structure: the right $\mathcal{O}$-module and right $\mathcal{O}$-comodule structures are given by $\operatorname{Id}_{A} \otimes m_{\mathcal{O}}$ and $d=\operatorname{Id}_{A} \otimes \Delta_{\mathcal{O}}$, where $m_{\mathcal{O}}$ and $\Delta_{\mathcal{O}}$ are the multiplication and comultiplication of $\mathcal{O}$, respectively [14, Examples 10.3, 10.4]. Then $A_{\mathcal{O}} \hookrightarrow A_{\mathcal{O}} \otimes \mathcal{O} \hookrightarrow \operatorname{Hom}_{\mathbb{k}}\left(H, A_{\mathcal{O}}\right)$ via $d$, with $(d b)(h)=h \rightarrow b$ for $b \in A_{\mathcal{O}}$. Now let $V \subseteq A_{\mathcal{O}}$ be a $\mathbb{k}$-subspace that is stable under right multiplication by $\mathcal{O}$ and under (8). Then $d V \subseteq \operatorname{Hom}_{\mathbb{k}}(H, V) \cap\left(A_{\mathcal{O}} \otimes \mathcal{O}\right)=V \otimes \mathcal{O}$ and so $V$ is a $\mathcal{O}$-Hopf submodule of $A_{\mathcal{O}}$. By the Structure Theorem for Hopf modules [14, $\left.\S 10.1 .2\right], V$ is generated as right $\mathcal{O}$-module by the subspace of $\mathcal{O}$-coinvariants, $V^{\text {co } \mathcal{O}}=\{v \in V \mid \delta v=v \otimes 1\}$. Since $V^{\operatorname{co} \mathcal{O}} \subseteq\left(A_{\mathcal{O}}\right)^{\operatorname{co} \mathcal{O}}=A \otimes \mathcal{O}^{\operatorname{co} \mathcal{O}}=A \otimes \mathbb{k}$, it follows that $V=W \otimes \mathcal{O}$ with $W=V^{\operatorname{co} \mathcal{O}}$.

\subsection{Coefficient Hopf algebras of locally finite actions.}

1.3.1. Assume that the action $H C A$ is locally finite, that is, $A=A_{\text {fin }}$. Then, since $\delta: A \rightarrow$ $(B, \rightarrow)$ is a morphism in ${ }_{H} \mathrm{Alg}(\S 1.1)$, the image $\delta A$ is contained in $(B, \rightarrow)_{\text {fin }}=A \otimes H^{\circ}$ and $A$ becomes a right $H^{\circ}$-comodule algebra via $\delta$ [14, Proposition 10.26]. Any Hopf subalgebra $\mathcal{O} \subseteq H^{\circ}$ such that $\delta A$ is contained in the subalgebra $A_{\mathcal{O}}=A \otimes \mathcal{O} \subseteq(B,-)_{\text {fin }}$ will be called a coefficient Hopf algebra for $H \subset A$. We then have the following version of (2), which makes $A$ a right $\mathcal{O}$-comodule algebra:

$$
\begin{aligned}
\delta: A & \longrightarrow A_{\mathcal{O}} \\
a & \longmapsto a_{0} \otimes a_{1}
\end{aligned} \quad \text { with } \quad h . a=a_{0}\left\langle a_{1}, h\right\rangle \quad(h \in H, a \in A) .
$$


Any coefficient Hopf algebra for $H C A$ will also serve as a coefficient Hopf algebra for the $H$-action on quotients of $A$ modulo $H$-ideals and on $H$-module subalgebras of $A$, and the intersection of all coefficient Hopf subalgebras for $H C A$ is the unique smallest one.

Lemma 5. Let $H \subset A$ be locally finite and let $\mathcal{O} \subseteq H^{\circ}$ be a coefficient Hopf algebra. Then:

(a) All subspaces of $A_{\mathcal{O}}$ of the form $W \otimes \mathcal{O}$, where $W \subseteq A$ is an $H$-stable $\mathbb{k}$-subspace, are stable under the maps $\Phi, \Psi(\S 1.1)$.

(b) If $I$ is an ideal of $A$, then $I: H=\Psi(I \otimes \mathcal{O}) \cap A$.

Proof. (a) If $W \subseteq A$ is $H$-stable, then $\delta W \subseteq W \otimes \mathcal{O}$. Thus, for any $a \in W$ and $f \in \mathcal{O}$, right $\mathcal{O}$-linearity of $\Phi$ and (6) now give $\Phi(a \otimes f)=\delta(a) f=a_{0} \otimes a_{1} f \in W \otimes \mathcal{O}$. Similarly, $\Psi(a \otimes f)=a_{0} \otimes \mathrm{S}^{*}\left(a_{1}\right) f \in W \otimes \mathcal{O}$, proving stability of $W \otimes \mathcal{O}$ under $\Phi$ and $\Psi$.

(b) By (1), $I: H=\delta^{-1}\left(\operatorname{Hom}_{\mathfrak{k}}(H, I)\right)=\delta^{-1}(I \otimes \mathcal{O})$. Putting $I^{\prime}=\Psi(I \otimes \mathcal{O})$ and using the identity $\Phi \circ \iota=\delta$ from (6), we obtain $I: H=\delta^{-1}(I \otimes \mathcal{O})=(\iota)^{-1}\left(I^{\prime}\right)=I^{\prime} \cap A$.

1.3.2. The action $H C A$ is certainly locally finite if the $H$-module algebra $A$ under consideration is generated as $\mathbb{k}$-algebra by a finite-dimensional $H$-stable subspace $V \subseteq A$. Assume this to be the case and let $\rho: H \rightarrow \operatorname{End}_{\mathfrak{k}}(V)$ denote the algebra map given by the operation of $H$ on $V$. Fixing a $\mathbb{k}$-basis $\left(v_{i}\right)_{1}^{n}$ of $V$ and letting $\left(v_{i}^{*}\right)$ denote the dual basis of $V^{*}$, we obtain the basis $\left(v_{i} \otimes v_{j}^{*}\right)$ of $\operatorname{End}_{\mathbb{k}}(V) \cong V \otimes V^{*}$ and linear forms $\rho_{i, j}$ such that

$$
\rho h=\sum_{i, j} v_{i} \otimes v_{j}^{*}\left\langle\rho_{i, j}, h\right\rangle \quad(h \in H) .
$$

The isomorphism $\operatorname{End}_{\mathbb{k}}(V) \cong \mathrm{M}_{n}(\mathbb{k})$ given by our choice of basis for $V$ allows us to write $\rho=\left(\rho_{i, j}\right): H \rightarrow \mathrm{M}_{n}(\mathbb{k}):$ the scalar $\left\langle\rho_{i, j}, h\right\rangle \in \mathbb{k}$ is the $(i, j)$-entry of the matrix $\rho h$. Thus, $\rho_{i, j} \in H^{\circ}$, because $\rho_{i, j}$ vanishes on the cofinite ideal $\operatorname{Ker} \rho$, and $\Delta \rho_{i, j}=\sum_{k} \rho_{i, k} \otimes \rho_{k, j}$. On the generating subspace $V \subseteq A$, the algebra map (10) takes the form

$$
\delta v=\sum_{i, j} v_{i}\left\langle v_{j}^{*}, v\right\rangle \otimes \rho_{i, j} \quad(v \in V)
$$

and we may take $\mathcal{O}$ to be the Hopf subalgebra of $H^{\circ}$ that is generated by the matrix coefficient functions $\rho_{i, j}$. If $H$ is involutory, then $\mathcal{O}$ is the $\mathbb{k}$-subalgebra of $H^{\circ}$ that is generated by all $\rho_{i, j}$ and $\mathrm{S}^{*} \rho_{i, j}$. We will call $\mathcal{O}$ the coefficient Hopf algebra of the representation $V \in \operatorname{Rep} H$; a more general situation is discussed in [14, Exercise 9.2.3].

\section{The COCOMMUTATIVE CASE}

\subsection{The $\cdot$-action.}

2.1.1. We recall some general ring-theoretic material that will be tacitly used in the next proposition and throughout the remainder of this article. A ring homomorphism $f: R \rightarrow S$ is called centralizing if the ring $S$ is generated by the image $f R$ and its centralizer in $S$. If $f$ makes $S$ a free $R$-module having a basis that centralizes $f R$, then $f$ is called free centralizing. Any centralizing homomorphism $f$ restricts to a map of centers, $\mathcal{Z} R \rightarrow \mathcal{Z} S$; the assignment $I \mapsto(f I) S$ sends (two-sided) ideals of $R$ to ideals of $S$; and $P \mapsto f^{-1} P$ gives a well-defined $\operatorname{map} \operatorname{Spec} S \rightarrow \operatorname{Spec} R[11, \S 1.5]$. 
2.1.2. The proposition below shows that, for $H$ cocommutative, we may view $B$ and various subalgebras of $B$ as $H$-module algebras with action (5), which we will refer to as the -action, rather than the $\rightarrow$-action (4). When using the latter $H$-action, we will continue write $(B, \rightarrow)$; otherwise, the $\cdot$-action is assumed.

Proposition 6. Let $H$ be cocommutative and let $\mathcal{O} \subseteq H^{\circ}$ be a coefficient Hopf algebra for the action $H C A_{\text {fin }}$. Then:

(a) $\Phi, \Psi$ are algebra automorphisms of $B$ stabilizing the subalgebra $A_{\text {fin, } \mathcal{O}}:=A_{\text {fin }} \otimes \mathcal{O}$.

(b) $B \in{ }_{H} \mathrm{Alg}$ with the - -action and $B^{H}=\Psi A$.

(c) $A_{\mathcal{O}}$ is an $H$-module subalgebra of $B$, with $\left(A_{\mathcal{O}}\right)_{\mathrm{fin}}=A_{\mathrm{fin}, \mathcal{O}}$ and $\left(A_{\mathcal{O}}\right)^{H}=\Psi\left(A_{\mathrm{fin}}\right)$. The inclusion $\left(A_{\mathcal{O}}\right)^{H} \subseteq\left(A_{\mathcal{O}}\right)_{\text {fin }}$ is free centralizing.

(d) The following maps are bijections that are inverse to each other:

$$
\begin{gathered}
\left\{\text { ideals of } A_{\text {fin }}\right\} \stackrel{\sim}{\longleftarrow}\left\{H \text {-ideals of } A_{\text {fin }, \mathcal{O}}\right\} \stackrel{\sim}{\longleftarrow}\left\{\text { ideals of }\left(A_{\mathcal{O}}\right)^{H}\right\} \\
I \longmapsto I^{\prime}:=\Psi(I \otimes \mathcal{O}) \longmapsto I^{\prime} \cap\left(A_{\mathcal{O}}\right)^{H} \\
\Phi\left(J^{\prime \prime}\right) \cap A_{\text {fin }} \longleftrightarrow J^{\prime \prime}:=J A_{\text {fin }, \mathcal{O}} \longleftrightarrow J
\end{gathered}
$$

Proof. (a) For $b, b^{\prime} \in B$ and $h \in H$, one computes using cocommutativity,

$$
\begin{aligned}
\Phi\left(b b^{\prime}\right)(h) & =h_{1} \cdot\left(b\left(h_{2}\right) b^{\prime}\left(h_{3}\right)\right)=\left(h_{1} \cdot b\left(h_{3}\right)\right)\left(h_{2} \cdot b^{\prime}\left(h_{4}\right)\right) \\
& =\left(h_{1} \cdot b\left(h_{2}\right)\right)\left(h_{3} \cdot b^{\prime}\left(h_{4}\right)\right)=\left(\Phi(b) \Phi\left(b^{\prime}\right)\right)(h) .
\end{aligned}
$$

Thus $\Phi$ is multiplicative and hence it is an algebra automorphisms of $B$; likewise for $\Psi=$ $\Phi^{-1}$. Stability of the subalgebra $A_{\text {fin, } \mathcal{O}}$ under $\Phi$ and $\Psi$ follows from Lemma 5(a) for $A_{\text {fin }}$.

(b) Since $\Psi$ is an algebra automorphism of $B$, the intertwining formula (7) together with the fact that $(B, \rightarrow) \in{ }_{H} \mathrm{Alg}$ implies that $B=(B, \cdot) \in{ }_{H} \mathrm{Alg}$ as well. Also by (7), $\Psi$ yields an algebra isomorphism $(B, \rightarrow)^{H} \cong B^{H}$ and so Lemma 3(a) gives the isomorphism $\Psi \circ \iota: A \stackrel{\sim}{\longrightarrow}(B, \rightarrow)^{H} \stackrel{\sim}{\longrightarrow} B^{H}$. Identifying $A$ with $\iota A=A \otimes \varepsilon$ as usual, we obtain the claimed equality $B^{H}=\Psi A$.

(c) The subalgebra $A_{\mathcal{O}} \subseteq B$ is stable under the -action by (9) and so it is an $H$-module subalgebra of $B$. The equality $\left(A_{\mathcal{O}}\right)_{\text {fin }}=A_{\text {fin, } \mathcal{O}}$ follows from [10, Corollary 6], because the $H$-action $\rightarrow$ on $\mathcal{O}$ is locally finite. Since $\mathrm{S}$ is an involution of $H$, the formula $(\Psi a)(h)=$ $\mathrm{S}\left(h_{1}\right) \cdot a\left\langle\varepsilon, h_{2}\right\rangle=\mathrm{S}(h) . a(a \in A, h \in H)$ shows that $\Psi a$ vanishes on some cofinite ideal of $H$ if and only if $a \in A_{\text {fin }}$. In that case, $\Psi a \in A_{\text {fin, } \mathcal{O}} \subseteq A_{\mathcal{O}}$ by (a). Thus, in view of part (b) and Lemma 3(b), $\Psi\left(A_{\text {fin }}\right)=B^{H} \cap(B,-)_{\text {fin }}=\left(A_{\mathcal{O}}\right)^{H}$ as asserted. Finally, since $\left(A_{\mathcal{O}}\right)_{\text {fin }}=A_{\text {fin }, \mathcal{O}}=\Psi\left(A_{\text {fin }, \mathcal{O}}\right)$ is generated by the commuting subalgebras $\Psi\left(A_{\text {fin }}\right)=\left(A_{\mathcal{O}}\right)^{H}$ and $\Psi \mathcal{O}$, with $\Psi \mathcal{O}$ providing an $\left(A_{\mathcal{O}}\right)^{H}$-basis of $\left(A_{\mathcal{O}}\right)_{\text {fin }}$, the inclusion $\left(A_{\mathcal{O}}\right)^{H} \subseteq\left(A_{\mathcal{O}}\right)_{\text {fin }}$ is free centralizing.

(d) Let $J$ be an ideal of $\left(A_{\mathcal{O}}\right)^{H}$. Since the inclusion $\left(A_{\mathcal{O}}\right)^{H} \subseteq\left(A_{\mathcal{O}}\right)_{\text {fin }}=A_{\text {fin, } \mathcal{O}}$ is centralizing by (c), $J A_{\text {fin, } \mathcal{O}}$ is an ideal of $A_{\text {fin, } \mathcal{O}}$, clearly an $H$-ideal. Further, $J A_{\text {fin }, \mathcal{O}} \cap\left(A_{\mathcal{O}}\right)^{H}=J$ by freeness of $A_{\text {fin, } \mathcal{O}}$ over $\left(A_{\mathcal{O}}\right)^{H}$. Now let $L$ be any $H$-ideal of $A_{\text {fin, } \mathcal{O}}$. Then $\Phi L$ is an ideal of $A_{\text {fin, } \mathcal{O}}$ that is stable for the - -action by (7). Thus, Lemma 4 implies that $\Phi L=I \otimes \mathcal{O}$ for some ideal $I$ of $A_{\text {fin }}$. Therefore, $L=\Psi(I \otimes \mathcal{O})=\Psi I \Psi \mathcal{O}=(\Psi I) A_{\text {fin, } \mathcal{O}}$ with $\Psi I$ being an ideal of $\left(A_{\mathcal{O}}\right)^{H}=\Psi\left(A_{\text {fin }}\right)$. This proves that extension and contraction give inverse bijections between the sets of ideals of $\left(A_{\mathcal{O}}\right)^{H}$ and $H$-ideals of $A_{\text {fin, } \mathcal{O}}$. The bijection between the sets of ideals of $\left(A_{\mathcal{O}}\right)^{H}$ and of $A_{\text {fin }}$ is a consequence of the equality $\Psi\left(A_{\text {fin }}\right)=\left(A_{\mathcal{O}}\right)^{H}$. 


\subsection{Integral actions.}

2.2.1. A locally finite action $H C A$ will be called integral if it has a coefficient Hopf algebra that is a commutative integral domain. Even though $H$ need not a priori be cocommutative, any integral $H$-action factors through a cocommutative Hopf quotient of $H$.

Proposition 7. Let $H C A$ be integral and $\mathbb{~} \mathrm{k}$ algebraically closed. Then $P: H$ is prime for every $P \in \operatorname{Spec} A$. Furthermore, $H$-Spec $A$ is the set of all prime $H$-ideals of $A$.

Proof. Let $\mathcal{O} \subseteq H^{\circ}$ be a coefficient Hopf algebra of the action $H \subset A$ that is an integral domain, and assume that $H$ is cocommutative, as we may. Then $\Psi$ is an algebra automorphism of $B$ that restricts to an automorphism of the subalgebra $A_{\mathcal{O}}$ (Proposition 6). For any $P \in$ Spec $A$, the ideal $P \otimes \mathcal{O}$ of $A_{\mathcal{O}}$ is prime [14, Lemma 11.19], and hence

$$
P^{\prime}:=\Psi(P \otimes \mathcal{O}) \in \operatorname{Spec} A_{\mathcal{O}} .
$$

By Lemma 5(b), $P: H=P^{\prime} \cap A$, which is a prime ideal of $A$, because $A \hookrightarrow A_{\mathcal{O}}$ is centralizing. The final assertion also follows, because the map $\operatorname{Spec} A \rightarrow H$-Spec $A, P \mapsto P: H$, is surjective for any locally finite action $H C A$ [14, Exercise 10.4.4].

2.2.2. Returning to the situation considered in $\S 1.3 .2$, assume that $A$ is affine, generated by an $H$-stable subspace $V \subseteq A$ with $n=\operatorname{dim}_{k} V<\infty$. We use our earlier notation, but we now also assume that $H$ is cocommutative and so involutory. The $\mathbb{k}$-subalgebra $\mathcal{O} \subseteq H^{\circ}$ that is generated by the functions $\rho_{i, j}$ and $\mathrm{S}^{*} \rho_{i, j}$ is thus a coefficient Hopf algebra for $H \subset A$.

Example 8 (Group algebras). Let $H=\mathbb{k} G$ be a group algebra. Then $H^{*}$ is the algebra $\mathbb{k}^{G}$ of all functions $G \rightarrow \mathbb{k}$ with pointwise addition and multiplication. The subalgebra $H^{\circ}$ is commonly referred to as the algebra of representative functions on $G$ and denoted by $R_{\mathbb{k}}(G)$ (e.g., [8, Chapter 1]). For any $g \in G$, the determinant $\operatorname{det} \rho g$ is nonzero and $\left\langle\mathrm{S}^{*} \rho_{i, j}, g\right\rangle=$ $\left\langle\rho_{i, j}, g^{-1}\right\rangle=\frac{1}{\operatorname{det} \rho g}\left\langle c_{j, i}, g\right\rangle$, where $\left\langle c_{j, i}, g\right\rangle$ denotes the $(j, i)$-cofactor of the matrix $\rho g \in$ $\mathrm{GL}_{n}(\mathbb{k})$, a polynomial in the entries of $\rho g$. Thus, $c_{j, i} \in R:=\mathbb{k}\left[\rho_{i, j} \mid i, j=1, \ldots, n\right]$ and $\mathrm{S}^{*} \rho_{i, j}=\frac{c_{j, i}}{\operatorname{det} \rho} \in R\left[(\operatorname{det} \rho)^{-1}\right]$, the subalgebra of $H^{\circ}$ that is generated by the functions $\rho_{i, j}$ and $(\operatorname{det} \rho)^{-1}=\mathrm{S}^{*} \operatorname{det} \rho$. We obtain

$$
\mathcal{O}=R\left[(\operatorname{det} \rho)^{-1}\right]=\mathbb{k}\left[(\operatorname{det} \rho)^{-1}, \rho_{i, j} \mid i, j=1, \ldots, n\right] .
$$

Now let $\mathbb{k}$ be algebraically closed. The group $\mathrm{GL}_{n}(\mathbb{k})$ is affine algebraic, with associated Hopf algebra $\mathcal{O}\left(\mathrm{GL}_{n}\right)$ as in [14, Example 9.19]. The Hopf algebra $\mathcal{O}$ is the image of $\mathcal{O}\left(\mathrm{GL}_{n}\right)$ under restriction to $\rho G \leq \mathrm{GL}_{n}(\mathbb{k})$. Finally, $\mathcal{O}$ is a domain if and only if $\rho G$ is an irreducible subset of $\mathrm{GL}_{n}(\mathbb{k})$ in the Zariski topology or, equivalently, the closure $\overline{\rho G}$ is a connected affine algebraic group.

Example 9 (Enveloping algebras). Let $\mathfrak{g}$ be a Lie $\mathbb{k}$-algebra and $H=U \mathfrak{g}$ its enveloping algebra. If char $\mathbb{k}=0$, then $H^{*}$ is a (commutative) domain; see [4, Chap. II, $\S 1, \mathrm{n}^{\circ} 5$ ] or Section 4.3 below. Therefore, the subalgebra $H^{\circ}$ and all coefficient Hopf algebras $\mathcal{O}$ are integral domains as well in characteristic 0 . The situation is different for char $\mathbb{k}=p>0$. Indeed, in this case, $\left\langle f^{p}, \mathfrak{g}\right\rangle=0$ for any $f \in H^{*}$. If $f \in H^{\circ}$ is grouplike or, equivalently, an algebra map, then so is $f^{p}$ and hence Ker $f^{p}$ is the ideal of $H$ that is generated by $\mathfrak{g}$. Therefore, $f^{p}=\varepsilon$ and so $(f-\varepsilon)^{p}=0$. If $\mathfrak{g} \neq[\mathfrak{g}, \mathfrak{g}]$ then we may choose $f \neq \varepsilon$, giving a nonzero nilpotent element of $H^{\circ}$. 


\subsection{The symmetric ring of quotients and the extended center.}

2.3.1. We briefly recall some background material on the symmetric ring of quotients $Q R$ of an arbitrary ring $R$ and its center, $\mathcal{C} R:=\mathcal{Z}(\mathrm{Q} R)$, the so-called extended center of $R$. See [14, Appendix E] for details. The ring $R$ is a subring of $\mathrm{Q} R$ and $\mathcal{C} R$ coincides with the centralizer of $R$ in $\mathrm{Q} R$. In particular, $\mathcal{Z} R \subseteq \mathcal{C} R$. If $\mathcal{Z} R=\mathcal{C} R$, then $R$ is called centrally closed. In general, we may consider the following subring of $\mathrm{Q} R$, possibly strictly larger than $R$ :

$$
\widetilde{R}:=R(\mathcal{C} R) \subseteq \mathrm{Q} R .
$$

If $R$ is semiprime, then $\widetilde{R}$ is a centrally closed ring [2, Theorem 3.2], called the central closure of $R$. If $R$ is a $\mathbb{k}$-algebra, then so is $\widetilde{R}$, because $\mathcal{Z} R \subseteq \mathcal{C} R=\mathcal{Z} \widetilde{R}$.

2.3.2. The next lemma concerns the extension of a given action $H C A$ to an action on $\mathrm{Q} A$. Part (a), which summarizes known facts, shows that this is always possible, in a unique way, in the situation that we are interested in. Indeed, any cocommutative Hopf algebra over an algebraically closed field is pointed [19, Lemma 8.0.1]. However, local finiteness of an action may be lost in the process. For instance, consider the action of the group $\mathbb{k}^{\times}$on the polynomial algebra $A=\mathbb{k}[x]$ that is determined by $\lambda . x=\lambda x$ for $\lambda \in \mathbb{k}^{\times}$and the extended action on the field $\mathrm{Q} A=$ Fract $A=k(x)$. If $\mathbb{k}$ is infinite, then $\mathbb{k}(x)_{\text {fin }}=\mathbb{k}\left[x^{ \pm 1}\right]$. Part (b) of the lemma focuses on the action $H C(\mathrm{Q} A)_{\text {fin }}$ rather than $H C \mathrm{Q} A$.

Lemma 10. Let $H$ be pointed cocommutative. Then:

(a) The $H$-action on $A$ extends uniquely to an action $H C \mathrm{C} A$ and this action stabilizes the subalgebras $\widetilde{A}=A(\mathcal{C} A)$ and $\mathcal{C} A$.

(b) If the action $H \subset A$ is locally finite with coefficient Hopf algebra $\mathcal{O}$, then the extended action $H \mathrm{C}(\mathrm{Q} A)_{\text {fin }}$ also has coefficient Hopf algebra $\mathcal{O}$.

Proof. (a) Since $H$ is pointed, [16, Cor. 3.5] tells us that the $H$-action on $A$ extends uniquely to an action on $\mathrm{Q} A$. This action stabilizes the center, $\mathcal{C} A$, because $H$ is cocommutative [5, Prop. 4]. Therefore, $\widetilde{A}$ is stable as well.

(b) Put $R=(\mathrm{Q} A)_{\text {fin }}$. So $A \subseteq R$ and the action $H \subset \mathrm{Q} A$ restricts to a locally finite action $H C R$ extending the $H$-action on $A$. We must show that the map $\delta: R \rightarrow R \otimes H^{\circ}$ (§1.3) has image in $R_{\mathcal{O}}=R \otimes \mathcal{O}$, given that $\delta A \subseteq A_{\mathcal{O}}$. Fix $r \in R$ and consider the subspace $V=H . r \subseteq R$, which is finite dimensional and $H$-stable. Therefore, the ideal $I=\{a \in A \mid a A V \subseteq A\}$ of $A$ has zero (left and) right annihilator in $R$ [14, Proposition E.1] and $I$ is an $H$-ideal as is readily verified using the following standard identity for $H$-module algebras:

$$
(h . x) y=h_{1} .\left(x\left(\mathrm{~S}\left(h_{2}\right) . y\right)\right) \quad(h \in H, x, y \in R) .
$$

By Lemma 5(a), $\Phi(I \otimes \mathcal{O})=I \otimes \mathcal{O}$. Furthermore, by Proposition 6(a), $\Phi$ gives an algebra automorphism of $R \otimes H^{\circ}$ stabilizing $A_{\mathcal{O}}$ and satisfying $\Phi \circ \iota=\delta$ by (6). Therefore,

$$
(I \otimes \varepsilon) \delta V \subseteq \Phi((I \otimes \mathcal{O})(V \otimes \varepsilon))=\Phi(I V \otimes \mathcal{O}) \subseteq \Phi(A \otimes \mathcal{O})=A_{\mathcal{O}} .
$$

Thus, $(I \otimes \varepsilon) \delta r \subseteq R_{\mathcal{O}}$. Since $\delta r \in R \otimes H^{\circ}$ and $I$ has zero right annihilator in $R$, it follows that $\delta r \in R_{\mathcal{O}}$, as desired. 


\section{PRIME STRATA}

3.1. Prime correspondences. The central closure $\widetilde{R}$ of a prime ring $R$ is also prime and its center, the extended center $\mathcal{C} R$, is a field. Thus, for an arbitrary ring $R$, we may associate to any $P \in \operatorname{Spec} R$ the field $\mathcal{C}(R / P)$, called the heart of $P$.

Proposition 11. Let $R$ be a centrally closed prime $\mathbb{k}$-algebra and put $K=\mathcal{C} R$. Let $S$ be any $K$-algebra and put $U=R \otimes_{K} S$. Then we have the following bijections, which are inverse to each other:

$$
\begin{array}{r}
\{P \in \operatorname{Spec} U \mid P \cap R=0\} \stackrel{\sim}{\sim} \operatorname{Spec} S \\
P \longmapsto P \cap S \\
U \mathfrak{p}=R \otimes_{K} \mathfrak{p} \longleftrightarrow \mathfrak{p}
\end{array}
$$

This correspondence preserves hearts: $\mathcal{C}(U / P) \cong \mathcal{C}(S / P \cap S)$. Moreover, if $U \in{ }_{H} \mathrm{Alg}$ with $S$ being an $H$-module subalgebra, then $H$-ideals are matched with $H$-ideals.

Proof. Apart from the last assertion, involving an $H$-action, the proposition is identical with [12, Proposition 5]. The bijections in the proposition, contraction and extension of ideals, both evidently send $H$-ideals to $H$-ideals in the given situation.

Proposition 12. Assume that $H$ is pointed cocommutative and let $R, T \in{ }_{H} \mathrm{Alg}$, with $R$ being prime. Put $V:=R \otimes T \subseteq \widetilde{V}:=\widetilde{R} \otimes T$, where $\widetilde{R}$ denotes the central closure of $R$. Then there is a bijection

$$
\begin{gathered}
\{P \in \operatorname{Spec} \widetilde{V} \mid P \cap \widetilde{R}=0\} \stackrel{\sim}{\sim}\{Q \in \operatorname{Spec} V \mid Q \cap R=0\} \\
P \longmapsto P \cap V
\end{gathered}
$$

This bijection is an order isomorphism for $\subseteq$ and it preserves hearts. Moreover, viewing $\widetilde{R} \in{ }_{H} \mathrm{Alg}$ with the extended $H$-action (Lemma 10) and $V, \widetilde{V} \in{ }_{H} \mathrm{Alg}$ with the standard $H$-action on tensor products, the bijection gives a bijection on $H$-stable primes.

Proof. Again, this proposition is covered by [12, Proposition 6] except for the statement about $H$-stability. To justify this assertion, note that tensor products of $H$-module algebras are again $H$-module algebras, with the standard $H$-action via $\Delta$, because $H$ is cocommutative. Thus, $\widetilde{V} \in{ }_{H} \mathrm{Alg}$ and all of $R, \widetilde{R}, T$ and $V$ are $H$-module subalgebras of $\widetilde{V}$. If $P$ is an $H$-ideal of $\widetilde{V}$, then its contraction, $P \cap V$, is clearly an $H$-ideal of $V$. Conversely, let $Q \in \operatorname{Spec} V$ with $Q \cap R=0$ be $H$-stable. In order to show that the preimage of $Q$ under the bijection in the proposition is an $H$-ideal of $\widetilde{V}$, we recall the construction of the preimage from [12]. The canonical epimorphism $\pi: V \rightarrow W:=V / Q$ is a map in ${ }_{H} \mathrm{Alg}$, and the restriction $\rho:=\left.\pi\right|_{R}: R \hookrightarrow W$ is an embedding of prime algebras and a centralizing map in ${ }_{H}$ Alg. By [12, Lemma 4], $\rho$ extends uniquely to an embedding of central closures, $\widetilde{\rho}: \widetilde{R} \hookrightarrow \widetilde{W}$, which is also centralizing.

Claim. $\widetilde{\rho}$ is a map in ${ }_{H} \mathrm{Alg}$ for the extended action $H \subset \widetilde{W}$ (Lemma 10).

To prove the claim, let $\widetilde{r} \in \widetilde{R}$ and $h \in H$. Fix a nonzero ideal $I$ of $R$ such that $(h . \widetilde{r}) I$ and all $\widetilde{r}\left(\mathrm{~S}\left(h_{2}\right) . I\right)$ are contained in $R$; this is possible by continuity of the action $H C R[16$, 
Proposition 2.2]. Using $H$-equivariance of $\rho$ one computes, with $x \in I$,

$$
\begin{aligned}
\widetilde{\rho}(h . \widetilde{r}) \rho x & =\rho((h \cdot \widetilde{r}) x)=\rho\left(h_{1} \cdot\left(\widetilde{r}\left(\mathrm{~S}\left(h_{2}\right) \cdot x\right)\right)\right)=h_{1} \cdot \rho\left(\widetilde{r}\left(\mathrm{~S}\left(h_{2}\right) \cdot x\right)\right) \\
& =h_{1} \cdot\left(\widetilde{\rho} \widetilde{r} \rho\left(\mathrm{S}\left(h_{2}\right) \cdot x\right)\right)=h_{1} \cdot\left(\widetilde{\rho} \widetilde{r}\left(\mathrm{~S}\left(h_{2}\right) \cdot \rho x\right)\right)=(h \cdot \tilde{\rho} \widetilde{r}) \rho x,
\end{aligned}
$$

where the last equality uses the identity (14). Thus, $(\widetilde{\rho}(h . \widetilde{r})-h . \widetilde{\rho} \widetilde{r}) \rho I=0$. Since $(\rho I) \widetilde{W}$ is a nonzero ideal of $\widetilde{W}$, it follows that $\widetilde{\rho}(h . \widetilde{r})=h . \widetilde{\rho} \widetilde{r}$, proving the claim.

The image $\pi T \subseteq W \subseteq \widetilde{W}$ centralizes $\rho R$ and hence also $\widetilde{\rho} \widetilde{R}=\rho R \widetilde{\rho}(\mathcal{Z} \widetilde{R})$, because $\widetilde{\rho}(\mathcal{Z} \widetilde{R}) \subseteq \mathcal{Z} \widetilde{W}$. Therefore, $\widetilde{\rho}$ and $\left.\pi\right|_{T}$ give a homomorphism $\widetilde{\pi}: \widetilde{V}=\widetilde{R} \otimes T \rightarrow \widetilde{W}$. The preimage of $Q$ in Proposition 12 is $\operatorname{Ker} \widetilde{\pi}$; see [12, proof of Proposition 6]. Finally, since $\widetilde{\rho}$ and $\left.\pi\right|_{T}$ are maps in ${ }_{H} \mathrm{Alg}$, it follows that $\tilde{\pi}$ is likewise. Hence Ker $\widetilde{\pi}$ is an $H$-ideal, as desired.

3.2. Proof of Theorem 1. Let $H$ be a cocommutative Hopf algebra over an algebraically closed field $\mathbb{k}$ and let $H \subset A$ be an integral action. Fix a coefficient Hopf algebra $\mathcal{O} \subseteq H^{\circ}$ that is an integral domain and write $A_{\mathcal{O}}=A \otimes \mathcal{O}$ as before. Given $I \in H$-Spec $A$, our goal is to describe the stratum $\operatorname{Spec}_{I} A$. Replacing $A$ by $A / I$, we may assume that $A$ is prime (Proposition 7) and focus on the set $\operatorname{Spec}_{0} A=\{P \in \operatorname{Spec} A \mid P: H=0\}$.

3.2.1. Put $X:=\left\{Q \in \operatorname{Spec} A_{\mathcal{O}} \mid Q \cap A=0\right\}$. We first establish a bijection between Spec $_{0} A$ and the subset $X^{H} \subseteq X$ consisting of all $Q \in X$ that are stable for the - -action (9). Recall that $\Psi$ gives an automorphism of the algebra $A_{\mathcal{O}}$ and, for any $P \in \operatorname{Spec} A$, we have $P^{\prime}:=\Psi(P \otimes \mathcal{O}) \in \operatorname{Spec} A_{\mathcal{O}}$; see Proposition 6 and (13). If $P \in \operatorname{Spec}_{0} A$, then $P^{\prime} \in X$ by Lemma 5(b). So we have a map $\operatorname{Spec}_{0} A \rightarrow X, P \mapsto P^{\prime}$, which is evidently injective. By Proposition 6(d), the image consists of $H$-ideals; so $P^{\prime} \in X^{H}$. Conversely, let $Q \in X^{H}$ be given and put $P=\Phi(Q) \cap A$; this is a prime ideal of $A$, because $\Phi(Q) \in \operatorname{Spec} A_{\mathcal{O}}$ and the embedding $A \hookrightarrow A_{\mathcal{O}}$ is centralizing. Also, Lemma 5(b) gives $P: H=P^{\prime} \cap A \subseteq Q \cap A=0$; so $P \in \operatorname{Spec}_{0} A$. Finally, $Q=P^{\prime}$ by Proposition 6(d). Thus, we have the desired bijection:

$$
\begin{gathered}
\operatorname{Spec}_{0} A \longrightarrow X^{H}=\left\{Q \in \operatorname{Spec} A_{\mathcal{O}} \mid Q \cap A=0 \text { and } Q \text { is stable under (9) }\right\} \\
P \longmapsto P^{\prime}=\Psi(P \otimes \mathcal{O})
\end{gathered}
$$

This bijection has the following properties, with (i) being evident and (ii) resulting from the algebra isomorphism $A_{\mathcal{O}} / P^{\prime} \cong A_{\mathcal{O}} /(P \otimes \mathcal{O}) \cong(A / P) \otimes \mathcal{O}$ that comes from $\Psi$ :

(i) $P_{1} \subseteq P_{2}$ if and only if $P_{1}^{\prime} \subseteq P_{2}^{\prime}$, and

(ii) $\mathcal{C}\left(A_{\mathcal{O}} / P^{\prime}\right) \cong \mathcal{C}((A / P) \otimes \mathcal{O})$.

3.2.2. Now put $K=\mathcal{C} A$ and $C=K \otimes \mathcal{O}$; the former is a $\mathbb{k}$-field and the latter a commutative integral domain, identical to the algebra $C_{0}$ in (3). Let $\widetilde{A}=A K$ denote the central closure of $A$ and put $\widetilde{A}_{\mathcal{O}}=\widetilde{A} \otimes \mathcal{O} \cong \widetilde{A} \otimes_{K} C$. Then we have the following isomorphisms of posets (for $\subseteq$ ), with $d_{1}$ coming from Proposition 12 and $d_{2}$ from Proposition 11:

$$
d: X=\left\{Q \in \operatorname{Spec} A_{\mathcal{O}} \mid Q \cap A=0\right\} \underset{d_{1}}{\sim}\left\{P \in \operatorname{Spec} \widetilde{A}_{\mathcal{O}} \mid P \cap \widetilde{A}=0\right\} \underset{d_{2}}{\sim} \operatorname{Spec} C .
$$


The composite of the bijection ': $\operatorname{Spec}_{0} A \stackrel{\longrightarrow}{\longrightarrow} X^{H}$ in $\S 3.2 .1$, the inclusion $X^{H} \hookrightarrow X$, and the above bijection $d=d_{2} \circ d_{1}$ yields the map

$$
c: \operatorname{Spec}_{0} A \underset{,}{\sim} X^{H} \longleftrightarrow X \underset{d}{\sim} \operatorname{Spec} C
$$

Since ' and both $d_{i}$ are order isomorphisms and preserve hearts, the same holds for $c$ :

(i) $c\left(P_{1}\right) \subseteq c\left(P_{2}\right)$ if and only if $P_{1} \subseteq P_{2}$, and

(ii) $\operatorname{Fract}(C / c(P))=\mathcal{C}(C / c(P)) \cong \mathcal{C}((A / P) \otimes \mathcal{O})$.

Finally, $\operatorname{Im} c$ consists exactly of the $H$-ideals in $\operatorname{Spec} C$. This amounts to checking that $Q \in$ $X$ is an $H$-ideal if and only if $d(Q) \in \operatorname{Spec} C$ is an $H$-ideal. But, by Lemma 10(a) and Proposition 6(c), $\widetilde{A}_{\mathcal{O}} \in{ }_{H}$ Alg for the $\cdot$-action, with $A_{\mathcal{O}}$ and $C=\mathcal{Z}\left(\widetilde{A}_{\mathcal{O}}\right)$ being $H$-module subalgebras. By Propositions 11 and 12 , we know that a given $P \in \operatorname{Spec} \widetilde{A}_{\mathcal{O}}$ with $P \cap \widetilde{A}=0$ is an $H$-ideal if and only if $d_{2}(P)=P \cap C \in \operatorname{Spec} C$ is an $H$-ideal and if and only if $d_{1}^{-1}(P)=P \cap A_{\mathcal{O}} \in X$ is an $H$-ideal. This completes the proof of Theorem 1 .

3.3. Tensor algebras. As an application of Theorem 1, we offer the following result on the tensor algebra $\mathrm{T} V$ of a finite-dimensional representation $V \in \operatorname{Rep} H$. The $H$-action on $V$ extends uniquely to an action $H C \mathrm{~T} V$ [14, 10.4.2].

Corollary 13. Let $H$ be a cocommutative Hopf algebra over an algebraically closed field $\mathbb{k}$ and let $V$ be a representation of $H$ with $2 \leq \operatorname{dim}_{\mathbb{k}} V<\infty$ and such that the coefficient Hopf algebra of $V(\S 1.3 .2)$ is a domain. Then every nonzero prime ideal of the tensor algebra TV contains a nonzero $H$-stable prime ideal.

Proof. The action $H C A=\mathrm{T} V$ is integral and we may apply Theorem 1 with $I=0$. By a result of Kharchenko [9], $\mathrm{Q} A=A$ and so $\mathcal{C} A=\mathcal{Z} A=\mathbb{k}$. Therefore, the algebra $C_{0}$ in Theorem 1 coincides with $\mathcal{O}$, the coefficient Hopf algebra of the representation $V$. Since $C_{0}=\mathcal{O}$ is $H$-simple by Lemma 4, we have $\operatorname{Spec}^{H} C_{0}=\{0\}$ and Theorem 1 gives $\operatorname{Spec}_{0} A=\{0\}$. Consequently, if $P$ is a nonzero prime ideal of $A$, then $P \notin \operatorname{Spec}_{0} A$ and so $P: H \neq 0$. Finally, $P: H$ is an $H$-stable prime ideal by Proposition 7 .

3.4. Torus actions. For rational torus actions, the set $\mathrm{Spec}^{H} C_{I}$ in Theorem 1 has a simpler description as $\operatorname{Spec} Z_{I}$ for an explicit commutative domain $Z_{I}$. This was shown in [13], but the presentation contains some inaccuracies which we will now repair.

To start with, let $H$ be pointed cocommutative and consider an arbitrary locally finite action $H \subset A$ with coefficient Hopf subalgebra $\mathcal{O} \subseteq H^{\circ}$. Associated to any $I \in H$-Spec $A$, we have the commutative algebra $C_{I}=\mathcal{C}(A / I) \otimes \mathcal{O} \in{ }_{H}$ Alg as in (3); the $H$-action on $C_{I}$ is (9) using the action $H \subset \mathcal{C}(A / I)$ from Lemma 10(a). This action need not be locally finite (§2.3.2). Following [13], we consider the locally finite part and the invariants:

$$
Z_{I}:=\mathcal{C}(A / I)_{\text {fin }} \supseteq F_{I}:=\mathcal{C}(A / I)^{H} .
$$

The invariant algebra $F_{I}$ is in fact a field [15, Lemma 1.4]. By Lemma 10(b), the action $H C Z_{I}$ has coefficient Hopf algebra $\mathcal{O}$ and Proposition 6(c) gives the following isomorphism which generalizes [13, Equation (2)]:

$$
Z_{I} \cong \Psi\left(Z_{I}\right)=\left(C_{I}\right)^{H} .
$$


Turning to torus actions, let $\mathbb{k}$ be algebraically closed and let $G$ be an algebraic $\mathbb{k}$-torus acting rationally on the $\mathbb{k}$-algebra $A$. As was remarked in $\S 0.4$, the algebra of polynomial functions $\mathcal{O}=\mathcal{O}(G)$ serves as a coefficient Hopf algebra for this action. Moreover, $\mathcal{O}$ is a Laurent polynomial algebra over $\mathbb{k}$, the algebra $C_{I}$ is a Laurent polynomial algebra over the field $\mathcal{C}(A / I)$, and the isomorphism $Z_{I} \cong\left(C_{I}\right)^{G}$ in (15) realizes $Z_{I}$ as a Laurent polynomial algebra over the field of $G$-invariants $F_{I}=\mathcal{C}(A / I)^{G}$; see the Stratification Theorem in [13] or [13, Equation (4)]. The set $\mathrm{Spec}^{H} C_{I}$ in Theorem 1 now is the set $\mathrm{Spec}^{G} C_{I}$ consisting of all $G$-stable prime ideals of $C_{I}$ and $\operatorname{Spec}\left(C_{I}\right)^{G} \cong \operatorname{Spec} Z_{I}$ by (15).

Lemma 14 (notation as above). Contraction and extension of ideals give a G-equivariant order isomorphism $\operatorname{Spec}^{G} C_{I} \stackrel{\sim}{\longrightarrow} \operatorname{Spec}\left(C_{I}\right)^{G}$.

Proof. By [13, Equation (5)], all $G$-stable ideals of $S:=C_{I}$ are generated by their intersection with $R:=S^{G}$. Therefore, the contraction map $\operatorname{Spec}^{G} S \rightarrow \operatorname{Spec} R, \mathfrak{P} \mapsto \mathfrak{P} \cap R$, is injective. If $\mathfrak{a}$ is an ideal of $R$, then its extension $\widetilde{\mathfrak{a}}:=\mathfrak{a} S$ is a $G$-stable ideal of $S$. Moreover, $\widetilde{\mathfrak{a}} \cap R=\mathfrak{a}$, because $S$ is free over $R$ by [13,2.3]. Thus, $\widetilde{\mathfrak{a}}$ is the only $G$-stable ideal of $S$ that contracts to $\mathfrak{a}$. Now let $\mathfrak{p} \in \operatorname{Spec} R$ be given and choose an ideal $\mathfrak{P}$ of $S$ maximal subject to the condition $\mathfrak{P} \cap R=\mathfrak{p}$. Then $\mathfrak{P}$ is prime and hence so is its $G$-core by Proposition 7. Thus, $\mathfrak{P}: G \in \operatorname{Spec}^{G} S$ and $\mathfrak{P}: G=\widetilde{\mathfrak{p}}$ by the foregoing. This proves surjectivity of the contraction map and that the inverse is given by extension. The statements about $G$-equivariance and preservation of inclusions in the lemma are clear.

\section{SEMIPRIMENESS}

4.1. Reformulations. We start this section by giving several reformulations, in terms of the semiprime radical operator $\sqrt{.}$, of the conclusion of Theorem 2 , which is equivalent to (ii) in the lemma below. The semiprime radical of a subset $X \subseteq A$, by definition, is the unique smallest semiprime ideal of $A$ containing $X$ :

$$
\sqrt{X}=\bigcap_{\substack{P \in \operatorname{Spec} A \\ P \supseteq X}} P .
$$

We continue to assume that $A \in{ }_{H} \mathrm{Alg}$; the Hopf algebra $H$ can be arbitrary for now.

Lemma 15. The following are equivalent:

(i) If $J$ is an $H$-ideal of $A$, then so is $\sqrt{J}$;

(ii) for all ideals $I$ of $A$, the $H$-core $\sqrt{I}: H$ is semiprime;

(iii) $H . \sqrt{I} \subseteq \sqrt{H . I}$ for any ideal I of $A$.

Proof. (i) $\Rightarrow$ (ii). We may assume that $I$ is semiprime. Then $\sqrt{I: H} \subseteq \sqrt{I}=I$, since $\sqrt{\text {. }}$ preserves inclusions. In fact, $\sqrt{I: H} \subseteq I: H$, because $\sqrt{I: H}$ is an $H$-ideal by (i). The reverse inclusion being trivial, it follows that $I: H=\sqrt{I: H}$ is semiprime.

(ii) $\Rightarrow$ (iii). Let $J$ denote the ideal of $A$ that is generated by the subset $H . I \subseteq A$. Then $\sqrt{I} \subseteq \sqrt{J}=\sqrt{H . I}$ and $J$ is easily seen to be an $H$-ideal. (If the antipode $\mathrm{S}$ is bijective, then $J=H . I$ [14, Exercise 10.4.3].) Thus, $J=J: H \subseteq \sqrt{J}: H$ and the latter ideal is semiprime by (ii). It follows that $\sqrt{J}=\sqrt{J: H} \subseteq \sqrt{J}: H$. Again, the reverse inclusion is clear; so $\sqrt{J}=\sqrt{J}: H$. Therefore, $H \cdot \sqrt{I} \subseteq H \cdot \sqrt{J}=\sqrt{J}=\sqrt{H . I}$.

(iii) $\Rightarrow$ (i). Specialize (iii) to the case where $I=J$ is an $H$-ideal. 
4.2. Extending the base field. For the proof of Theorem 2, we may work over an algebraically closed base field. This follows by taking $K$ to be an algebraic closure of $\mathbb{k}$ in the argument below.

Let $K / \mathbb{k}$ be any field extension and put $H^{\prime}=H \otimes K$ and $A^{\prime}=A \otimes K$. Then $A^{\prime} \in{ }_{H^{\prime}} \mathrm{Alg}$ and $H^{\prime}$ is cocommutative if $H$ is so. Assuming Theorem 2 to hold for $A^{\prime}$, our goal is to show that it also holds for $A$. So let $I$ be a semiprime ideal of $A$. Viewing $A$ as being contained in $A^{\prime}$ in the usual way, $I A^{\prime}$ is an ideal of $A^{\prime}$ satisfying $I A^{\prime} \cap A=I$. By Zorn's Lemma, we may choose an ideal $I^{\prime}$ of $A^{\prime}$ that is maximal subject to the condition $I^{\prime} \cap A=I$. Then $I^{\prime}$ is semiprime. For, if $J$ is any ideal of $A^{\prime}$ such that $J \supsetneqq I^{\prime}$, then $J \cap A \supsetneqq I$ by maximality of $I^{\prime}$, and so $(J \cap A)^{2} \nsubseteq I$ by semiprimeness of $I$. Since $(J \cap A)^{2} \subseteq J^{2} \cap A$, it follows that $J^{2} \nsubseteq I^{\prime}$, proving that $I^{\prime}$ is semiprime. Therefore, by our assumption, the core $I^{\prime}: H^{\prime}$ is semiprime. Since the extension $A \hookrightarrow A^{\prime}$ is centralizing, it follows that $\left(I^{\prime}: H^{\prime}\right) \cap A$ is a semiprime ideal of $A$. Finally, $\left(I^{\prime}: H^{\prime}\right) \cap A=\left\{a \in A \mid H^{\prime} . a \subseteq I^{\prime}\right\}=\left\{a \in A \mid H . a \subseteq I^{\prime} \cap A=I\right\}=I: H$ by (1), giving the desired conclusion that $I: H$ is semiprime.

\subsection{Enveloping algebras.}

4.3.1. For any ring $R$, let $R \llbracket X_{\lambda} \rrbracket_{\lambda \in \Lambda}$ denote the ring of formal power series in the commuting variables $X_{\lambda}(\lambda \in \Lambda)$ over $R$; see [3, Chap. III, $\left.\S 2, \mathrm{n}^{\circ} 11\right]$.

Lemma 16. Let $R$ be a ring, let $\Lambda$ be any set, and let $S$ be a subring of $R \llbracket X_{\lambda} \rrbracket_{\lambda \in \Lambda}$ such that $S$ maps onto $R$ under the homomorphism $R \llbracket X_{\lambda} \rrbracket_{\lambda \in \Lambda} \rightarrow R, X_{\lambda} \mapsto 0$. If $R$ is prime (resp., semiprime, a domain) then so is $S$.

Proof. We write monomials in the variables $X_{\lambda}$ as $X^{\boldsymbol{n}}=\prod_{\lambda} X_{\lambda}^{\boldsymbol{n}(\lambda)}(\boldsymbol{n} \in M)$, where $M=$ $\mathbb{Z}_{+}^{(\Lambda)}$ denotes the additive monoid of all functions $\boldsymbol{n}: \Lambda \rightarrow \mathbb{Z}_{+}$such that $\boldsymbol{n}(\lambda)=0$ for almost all $\lambda \in \Lambda$. Fix a total order $<$ on $M$ having the following properties (e.g., [1, Example 2.5]): every nonempty subset of $M$ has a smallest element; the zero function $\mathbf{0}$ is the smallest element of $M$; and $\boldsymbol{n}<\boldsymbol{m}$ implies $\boldsymbol{n}+\boldsymbol{r}<\boldsymbol{m}+\boldsymbol{r}$ for all $\boldsymbol{n}, \boldsymbol{m}, \boldsymbol{r} \in M$.

For any $0 \neq s=\sum_{\boldsymbol{n} \in M} s_{\boldsymbol{n}} X^{\boldsymbol{n}} \in R \llbracket X_{\lambda} \rrbracket_{\lambda \in \Lambda}$, we may consider its lowest coefficient, $s_{\text {min }}:=s_{\boldsymbol{m}}$ with $\boldsymbol{m}=\min \left\{\boldsymbol{n} \in M \mid s_{\boldsymbol{n}} \neq 0\right\}$. If $R$ is prime and $0 \neq s, t \in S$ are given, then $0 \neq s_{\min } r t_{\min }$ for some $r \in R$. By assumption, there exists an element $u \in S$ having the form $u=r+\sum_{\boldsymbol{n} \neq \mathbf{0}} u_{\boldsymbol{n}} X^{\boldsymbol{n}}$. It follows that $s u t \neq 0$, with $(s u t)_{\min }=s_{\min } r t_{\min }$. This proves that $S$ is prime. For the assertions where $R$ is semiprime or a domain, take $s=t$ or $r=1$, respectively.

4.3.2. Now let $H=U \mathfrak{g}$ be the enveloping algebra of an arbitrary Lie $\mathbb{k}$-algebra $\mathfrak{g}$ and assume that char $\mathbb{k}=0$. For the proof of the next proposition, we recall the structure of the convolution algebra $\operatorname{Hom}_{\mathbb{k}}(H, R)$ for an arbitrary $\mathbb{k}$-algebra $R$. Let $\left(e_{\lambda}\right)_{\lambda \in \Lambda}$ be a $\mathbb{k}$-basis of $\mathfrak{g}$ and fix a total order of the index set $\Lambda$. Put $M=\mathbb{Z}_{+}^{(\Lambda)}$ as in the proof of Lemma 16 and, for each $\boldsymbol{n} \in M$, put $e_{\boldsymbol{n}}=\prod_{\lambda}^{<} \frac{1}{\boldsymbol{n}(\lambda) !} e_{\lambda}^{\boldsymbol{n}(\lambda)} \in H$, where the superscript $<$ indicates that the factors occur in the order of increasing $\lambda$. The elements $e_{\boldsymbol{n}}$ form a $\mathbb{k}$-basis of $H$ by the PoincaréBirkhoff-Witt Theorem, and the comultiplication of $H$ is given by $\Delta e_{\boldsymbol{n}}=\sum_{\boldsymbol{r}+\boldsymbol{s}=\boldsymbol{n}} e_{\boldsymbol{r}} \otimes e_{\boldsymbol{s}}$; see [14, Example 9.5]. Writing $X^{\boldsymbol{n}}=\prod_{\lambda} X_{\lambda}^{\boldsymbol{n}(\lambda)}$ as in the proof of Lemma 16, we obtain an 
isomorphism of $\mathbb{k}$-algebras,

$$
\varphi: \operatorname{Hom}_{\mathbb{k}}(H, R) \stackrel{\sim}{\longrightarrow} R \llbracket X_{\lambda} \rrbracket_{\lambda \in \Lambda}, \quad f \mapsto \sum_{\boldsymbol{n} \in M} f\left(e_{\boldsymbol{n}}\right) X^{\boldsymbol{n}} .
$$

Under this isomorphism, the algebra map $u^{*}: \operatorname{Hom}_{\mathbb{k}}(H, R) \rightarrow R, f \mapsto f(1)$, coming from the unit map $u=u_{H}: \mathbb{k} \rightarrow H$ translates into the map $R \llbracket X_{\lambda} \rrbracket_{\lambda \in \Lambda} \rightarrow R, X_{\lambda} \mapsto 0$, as considered in Lemma 16.

The proposition below is not new; it can be found in Dixmier's book [6, 3.3.2 and 3.8.8], albeit with a very different proof. Recall that an ideal of a ring is said to be completely prime if the quotient is a domain.

Proposition 17. Let $H=U \mathfrak{g}$ be the enveloping algebra of a Lie $\mathbb{k}$-algebra $\mathfrak{g}$, let $A \in{ }_{H} \mathrm{Alg}$, and let $I$ be an ideal of $A$. Assume that char $\mathbb{k}=0$. If I is prime, semiprime or completely prime, then $I: H$ is likewise.

Proof. By (1), the core $I: H$ is identical to the kernel of the map $\delta_{I}: A \rightarrow \operatorname{Hom}_{\mathbb{k}}(H, A / I)$ that is given by $\delta_{I}(a)=(h \mapsto h . a+I)$. We need to show that the properties of being prime, semiprime, or a domain all transfer from $A / I$ to the subring $\delta_{I} A \subseteq \operatorname{Hom}(H, A / I)$ or, equivalently, to the subring $S \subseteq(A / I) \llbracket X_{\lambda} \rrbracket_{\lambda \in \Lambda}$ that corresponds to $\delta_{I} A$ under the above isomorphism $\varphi$. Consider the map $u^{*}: \operatorname{Hom}_{\mathbb{k}}(H, A / I) \rightarrow A / I, f \mapsto f(1)$, and note that $\left(u^{*} \circ \delta_{I}\right)(a)=a+I$ for $a \in A$. Therefore, $S$ maps onto $A / I$ under the map $(A / I) \llbracket X_{\lambda} \rrbracket_{\lambda \in \Lambda} \rightarrow$ $A / I, X_{\lambda} \mapsto 0$. Now all assertions follow from Lemma 16.

4.3.3. For an arbitrary cocommutative Hopf algebra $H$, we cannot expect a result as strong as Proposition 17: group algebras provide easy counterexamples to the primeness and complete primeness assertions. Indeed, let $H=\mathbb{k} G$ be the group $\mathbb{k}$-algebra of the group $G$ and let $A \in{ }_{H} \mathrm{Alg}$. Then $I: H=\bigcap_{g \in G} g . I$ for any ideal $I$ of $A$. If $I$ is semiprime, then so are all $g . I$, because each $g \in G$ acts on $A$ by algebra automorphisms, and hence $\bigcap_{g \in G} g . I$ will be semiprime also. However, primeness and complete primeness, while inherited by each g.I, are generally lost upon taking the intersection.

4.4. Proof of Theorem 2. Let $H$ be cocommutative Hopf and assume that char $\mathbb{k}=0$ and that $k$ is algebraically closed, as we may by $\S 4.2$. Then $H$ has the structure of a smash product, $H \cong U \# V$, where $U$ is the enveloping algebra of the Lie algebra of primitive elements of $H$ and $V$ is the group algebra of the group of grouplike elements of $H$; see $[19, \S 13.1]$ or [17, $\S 15.3]$. Thus, both $U$ and $V$ are Hopf subalgebras of $H$ and $H=U V$, the $\mathbb{k}$-space spanned by all products $u v$ with $u \in U$ and $v \in V$. Viewing $A \in{ }_{U}$ Alg and $A \in{ }_{V}$ Alg by restriction, repeated application of (1) gives the following equality for any ideal $I$ of $A$ :

$$
I: H=\{a \in A \mid U V . a \subseteq I\}=\{a \in A \mid V . a \subseteq I: U\}=(I: U): V .
$$

If $I$ is semiprime, then so is $I: U$ (Proposition 17). Our remarks on group algebras in the first paragraph of this proof further give semiprimeness of $(I: U): V$. Thus, $I: H$ is semiprime and Theorem 2 is proved.

Acknowledgment. We thank the referee for pointing out reference [6] in connection with Proposition 17 above. 


\section{REFERENCES}

[1] Matthias Aschenbrenner and Christopher J. Hillar, Finite generation of symmetric ideals, Trans. Amer. Math. Soc. 359 (2007), no. 11, 5171-5192. MR 2327026 (2008g:13030)

[2] W. E. Baxter and W. S. Martindale, III, Jordan homomorphisms of semiprime rings, J. Algebra 56 (1979), no. 2, 457-471. MR 528587 (80f:16008)

[3] Nicolas Bourbaki, Algèbre. Chapitres 1 à 3, Hermann, Paris, 1970. MR 43 \#2

[4] __ Groupes et algèbres de Lie. Chapitre II: Algèbres de Lie libres. Chapitre III: Groupes de Lie, Éléments de mathématique. Fasc. XXXVII., Hermann, Paris, 1972, Actualités Scientifiques et Industrielles, No. 1349. MR 58 \#28083a

[5] Miriam Cohen, Smash products, inner actions and quotient rings, Pacific J. Math. 125 (1986), no. 1, 45-66. MR 860749 (88a:16019)

[6] Jacques Dixmier, Enveloping algebras, Graduate Studies in Mathematics, vol. 11, American Mathematical Society, Providence, RI, 1996, Revised reprint of the 1977 translation. MR 1393197 (97c:17010)

[7] Kenneth R. Goodearl and Edward S. Letzter, The Dixmier-Moeglin equivalence in quantum coordinate rings and quantized Weyl algebras, Trans. Amer. Math. Soc. 352 (2000), no. 3, 1381-1403. MR 1615971 (2000j:16040)

[8] Gerhard P. Hochschild, Basic theory of algebraic groups and Lie algebras, Graduate Texts in Mathematics, vol. 75, Springer-Verlag, New York-Berlin, 1981. MR 620024 (82i:20002)

[9] Vladislav K. Kharchenko, Algebras of invariants of free algebras, Algebra i Logika 17 (1978), no. 4, 478487, 491. MR 538309

[10] Stefan Kolb, Martin Lorenz, Bach Nguyen, and Ramy Yammine, On the adjoint representation of a Hopf algebra, arXiv:1905.03020v2, 2019.

[11] Martin Lorenz, Group actions and rational ideals, Algebra Number Theory 2 (2008), no. 4, 467-499. MR 2411408 (2009i:16058)

[12] _ Algebraic group actions on noncommutative spectra, Transform. Groups 14 (2009), no. 3, 649-675. MR 2534802 (2010m:14060)

[13] _ On the stratification of noncommutative prime spectra, Proc. Amer. Math. Soc. 142 (2014), 30133017.

[14] A tour of representation theory, Graduate Studies in Mathematics, vol. 193, American Mathematical Society, Providence, RI, 2018.

[15] Jerzy Matczuk, Centrally closed Hopf module algebras, Comm. Algebra 19 (1991), no. 7, 1909-1918. MR 1121113 (92i:16031)

[16] Susan Montgomery, Bi-invertible actions of Hopf algebras, Israel J. Math. 83 (1993), no. 1-2, 45-71. MR 1239716 (94g:16047)

[17] David E. Radford, Hopf algebras, Series on Knots and Everything, vol. 49, World Scientific Publishing Co. Pte. Ltd., Hackensack, NJ, 2012. MR 2894855

[18] Serge Skryabin, Hopf algebra orbits on the prime spectrum of a module algebra, Algebr. Represent. Theory 13 (2010), no. 1, 1-31. MR 2585121 (2011e:16058)

[19] Moss E. Sweedler, Hopf algebras, Mathematics Lecture Note Series, W. A. Benjamin, Inc., New York, 1969. MR 0252485 (40 \#5705) 